\title{
What can we learn from a two-brain approach to verbal interaction?
}

\author{
Lotte Schoot ${ }^{\mathrm{a}, *}$, Peter Hagoort ${ }^{\mathrm{a}, \mathrm{b}}$, Katrien Segaert $^{\mathrm{c}}$ \\ a Max Planck Institute for Psycholinguistics, P.O. Box 310, 6500 AH Nijmegen, The Netherlands \\ ${ }^{\mathrm{b}}$ Donders Institute for Brain, Cognition and Behaviour, P.O. Box 9101, 6500 HB, Nijmegen, The Netherlands \\ ' School of Psychology, University of Birmingham, Edgbaston, Birmingham, B15 2TT, United Kingdom
}

\section{A R T I C L E I N F O}

\section{Article history:}

Received 30 March 2016

Received in revised form 10 June 2016

Accepted 12 June 2016

Available online 14 June 2016

\section{Keywords:}

Hyperscanning

Social neuroscience

Verbal communication

Mutual understanding

Alignment

\begin{abstract}
A B S T R A C T
Verbal interaction is one of the most frequent social interactions humans encounter on a daily basis. In the current paper, we zoom in on what the multi-brain approach has contributed, and can contribute in the future, to our understanding of the neural mechanisms supporting verbal interaction. Indeed, since verbal interaction can only exist between individuals, it seems intuitive to focus analyses on interindividual neural markers, i.e. between-brain neural coupling. To date, however, there is a severe lack of theoretically-driven, testable hypotheses about what between-brain neural coupling actually reflects. In this paper, we develop a testable hypothesis in which between-pair variation in between-brain neural coupling is of key importance. Based on theoretical frameworks and empirical data, we argue that the level of between-brain neural coupling reflects speaker-listener alignment at different levels of linguistic and extra-linguistic representation. We discuss the possibility that between-brain neural coupling could inform us about the highest level of inter-speaker alignment: mutual understanding.
\end{abstract}

(C) 2016 Elsevier Ltd. All rights reserved.

\section{Contents}

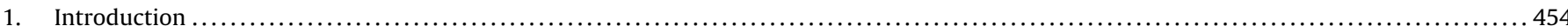

2. A multi-brain approach to studying the relationship between language comprehension and production........................455

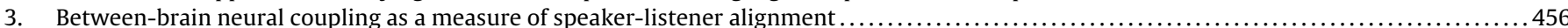

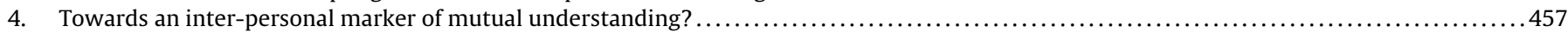

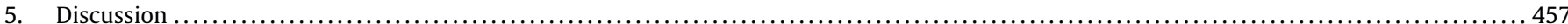

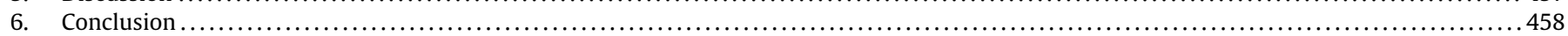

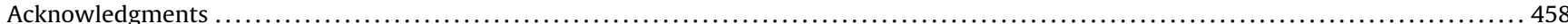

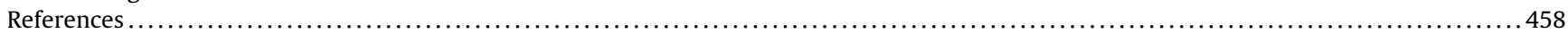

\section{Introduction}

Recent advances in the field of social neuroscience suggest that in order to get at a complete understanding of the different neural processes involved in social interaction, the dynamic interplay between the brains of two interacting individuals needs to be studied (e.g. Hari et al., 2015; Hasson et al., 2012). The inter-individual neural markers of interest are inter-subject correlations in temporal and spatial patterns of brain activity, also known as between-brain neural coupling (Stephens et al., 2010). Assessing the level of between-brain neural coupling requires mea-

* Corresponding author at: Max Planck Institute for Psycholinguistics, P.O. Box 310, 6500 AH Nijmegen, The Netherlands.

E-mail address: Lotte.Schoot@mpi.nl (L. Schoot). suring brain activity for two (or more) participants involved in a social interaction, a technique called hyperscanning (brain activation is measured for both participants at the same time) or pseudo-hyperscanning (measuring brain activity for both participants in the interaction, but sequentially, one participant at a time). Since the first application of the hyperscanning method in fMRI (Montague et al., 2002), it has been applied to other neuroimaging methods as well (EEG, fNIRS and MEG) and used to investigate different aspects of social interaction (for overviews see Babiloni and Astolfi, 2014; Dumas et al., 2011; Konvalinka and Roepstorff, 2012).

In the current paper, we zoom in on what the multi-brain approach has contributed, and can contribute in the future, to our understanding of verbal interaction. Given the fact that verbal interaction is ubiquitous in our everyday lives, it is surprising 
that relatively few multi-brain studies have focused on this specific form of social interaction. So far, most multi-brain verbal communication studies have used the hyperscanning method to investigate the spatial and temporal relationship between neural mechanisms which support language production by the speaker and comprehension by the listener (see Section 2). Although these studies claim to investigate the neural correlates of verbal information transfer, they generally ignore pair-specific information about the quality of the interaction: whether information transfer was actually successful. However, it has been previously suggested that successful communication or mutual understanding can be operationalized in the form of inter-subject correlations in brain activity (Menenti et al., 2012; Stephens et al., 2010). We argue that the reason this idea has not been investigated in more detail is that although intuitive, it is not backed up by a strong theoretical framework leading to testable hypotheses.

We will discuss a recent theoretical framework (Friston and Frith, 2015a, 2015b) leading to the testable hypothesis that the strength of between-brain neural coupling reflects speaker-listener alignment at multiple representational levels (Section 3). In Section 4 , we consider the possibility that between-brain neural coupling could reflect alignment at the highest representational level possible: the level of the situation model. If so, this would provide us with an inter-personal marker of successful communication. We discuss several possibilities to test this hypothesis before concluding this paper with an outlook on how the hyperscanning method may be used in future research.

\section{A multi-brain approach to studying the relationship between language comprehension and production}

There have been a few studies that have investigated speakerlistener neural coupling during verbal communication (Dikker et al., 2014; Jiang et al., 2012; Kuhlen et al., 2012; Silbert et al., 2014; Stephens et al., 2010). Like two-brain studies on non-verbal communication (Anders et al., 2011; Ménoret et al., 2014; Schippers et al., 2010), most of these studies have used the multi-brain approach to investigate 'information flow' from the brain of the sender (the speaker) to the brain of the receiver (the listener). In other words, to what extent is neural activity associated with encoding of information by the sender mirrored in the activity associated with the decoding of that information by the receiver? The reasoning here is as follows: if activity in area $\mathrm{X}$ in the brain of the sender is temporally correlated with activity in area $X$ in the brain of the listener (perhaps with a delay), this indicates that area $\mathrm{X}$ is associated with encoding as well as decoding of information. More specifically, for verbal communication, such a finding would indicate that the neural infrastructures for language production and comprehension at least in part overlap, opposing the classical Wernicke-Lichtheim-Geschwind model, in which a strict division of labor is proposed. However, speaker-listener correlations in brain activity would be in line with converging evidence from patient data (e.g. Caramazza and Zurif, 1976) and one-brain neuroimaging studies (Menenti et al., 2011; Segaert et al., 2012), which support the view that the same brain regions may support language production as well as comprehension.

In the first two-brain study on verbal communication, Stephens et al. (2010) recorded a speaker telling an unrehearsed real-life story and played this recording to eleven listeners. Crucially, brain activity was measured with fMRI for both the speaker and listeners. By modeling the expected activity in the listeners' brains based on the speaker's neural activity during speech production, Stephens et al. tested whether the neural activity of the speaker was temporally and spatially coupled to the shared neural activity observed across all listeners. In other words, they tested whether there was overlap in brain areas involved in producing and listening to speech, and whether these activation patterns in the speaker and listener's brains were temporally related to each other (e.g. whether the speaker's brain activity preceded the listener's brain activity). Indeed, Stephens et al. found widespread spatial coupling between brain activity in the speaker and listener, both in areas classically associated with language processing (such as the left superior temporal gyrus and the left inferior frontal gyrus), and in areas that support processes that are generally considered to be extra-linguistic (such as the precuneus and the medial prefrontal cortex). Temporally, for most (but not all) of these areas within the listeners' brains, activity lagged behind the speaker's brain by three to six seconds. Crucially, the spatial and temporal coupling that was found when the speaker and listeners processed the same story largely disappeared when listeners were listening to a Russian speaker, or when the brain activity of the speaker that was used to model the listeners' neural responses was associated with the speaker telling a different story than the story the listeners were listening to. This indicates that between-brain neural coupling does not only depend on producing and hearing the same acoustic signal, but also on the extent to which the signal can be decoded by the listener. If the listener cannot process the linguistic input to extract meaning and structure, the underlying linguistic processes do not match and there will thus not be any coupling in areas necessary for these processes.

Other fMRI studies in which the two-brain approach has been applied to similar verbal information transfer paradigms report similar results (Silbert et al., 2014; Spiegelhalder et al., 2014). In general, these studies report enhanced between-brain neural coupling during one-way communication; when producing or listening to the same verbal information stimulus, the brain activity of the speaker is reflected in the brain of the listener. Together, these studies provide a novel type of evidence in favor of the hypothesis that language production and comprehension depend (at least in part) on the same neural mechanisms. This information is crucial for theories trying to explain behavioral phenomena in dialogue which require close coupling between language production and comprehension processes and/or shared representations at different linguistic and non-linguistic levels (see also: Pickering and Garrod, 2014). One example of such a behavioral phenomenon in dialogue is syntactic priming: hearing a specific sentence structure increases the chance that speakers will use this structure in a subsequent utterance. For this type of behavioral priming to occur from comprehension to production, one must assume some degree of shared representation and/or processing at the level of sentence structure (Menenti et al., 2012).

Most multi-brain verbal interaction studies have thus used speaker-listener between-brain neural coupling to identify neural networks associated with language production as well as language comprehension. These results have been taken as evidence to support theories which propose that a certain degree of overlap in the neural networks underlying language production and comprehension is necessary to explain inter-personal behavioral phenomena in natural conversation, such as priming. However, we would also like to make a critical observation here. By focusing research on identifying brain networks required for language production and comprehension, most of the studies discussed above have reported between-brain neural coupling common for all interaction pairs in their sample. Indeed, by comparing inter-subject correlations in pairs that produce and understand the same communicative signal to the correlations in pairs who are not coupled in this way, one can extract brain areas that are necessary to produce the signal on the one hand, and comprehend it on the other. However, by focusing on what is present across all pairs, we lose pair-specific information about the quality of the interaction, which may vary from pair to pair. In the next section, we will discuss what between-pair vari- 
ation in speaker-listener neural coupling could tell us about the quality of verbal interaction.

\section{Between-brain neural coupling as a measure of speaker-listener alignment}

So far, we have discussed results of two-brain studies using verbal communication paradigms that have looked at betweenbrain neural coupling at the group level, identifying brain areas that show reliable inter-subject correlations across all real communication pairs. In this section, we will instead focus on variations in the level of between-brain neural coupling between different sets of communication pairs. More specifically, we hypothesize that between-pair differences in the extent of between-brain neural coupling may be explained by the level of alignment between speaker and listener at multiple levels of linguistic and extralinguistic representations.

Our hypothesis is largely based on a recent theoretical framework proposed by Friston and Frith (2015a, 2015b). As an extension of the more general predictive coding framework, Friston and Frith consider communication in terms of inferences about others. Indeed, predictive coding theory assumes that our brain infers the causes of sensory input to be able to correctly predict upcoming input. The predictive coding framework fits within a shift in cognitive neuroscience away from seeing the brain as a passive filter of information and towards a view of the brain as an active organ that generates predictions about upcoming sensory input. These top-down predictions are compared to representations at lower levels of the hierarchy to form a prediction error: a bottom-up signal reflecting the mismatch between prediction and actual sensory input. Prediction errors can be seen as feedback signals that ensure that the internal or generative model is updated, so that predictions are adapted and prediction errors for future incoming input are minimized.

In the predictive coding framework, the main goal of the brain is to minimize prediction error. According to Friston and Frith, prediction error for the listener in a communicative context would be minimized if they converge on a similar or identical internal or generative model as their partner. Put differently, alignment of these internal models would lead to successful predictions for the listener and thus facilitated communication. Crucially, Friston and Frith suggest that when the listener can correctly predict what the speaker will say next, their neural states will show what they call generalized synchrony. Friston and Frith explain generalized synchrony as knowing the neural state of one brain in a pair by knowing the neural state of the other brain in that pair. Indeed, this is very similar to the definition of between-brain neural coupling that we have used above: inter-subject correlations in brain activity.

But how is it that correctly predicting what the speaker says leads to coupling (generalized synchrony) between brain activity of speaker and listener? This would only be possible if speaking and listening are both driven by the same underlying processes. Indeed, this is what is proposed in the predictive coding framework: the predictions that are generated by any individual cannot only be tested against external input; they can also be enacted. According to Friston and Frith, action and perception (language production and comprehension) are two sides of the same coin. The predictions generated are amodal in nature and not specific for comprehension or production only. Therefore, when the listener has correctly inferred the speaker's generative model, their predictions will be similar, which is in turn reflected in generalized synchrony or between-brain neural coupling.

Generalized synchrony is a ubiquitous phenomenon in loosely coupled dynamical systems. In the context of communication and predictive coding, it attains a special status. This is because com- munication in the sense of aligning internal representations (i.e., a dialogue) requires turn taking and the reciprocal augmentation and attenuation of expressive versus receptive processes. If I can use my same predictive machinery to predict (and confirm) what I am listening to, as well as to provide motor predictions that allow me to articulate a narrative, then if we are in true alignment and are 'on the same page', then it does not matter whether you or I are speaking - because we should be hearing the same thing. This form of generalized synchrony can be regarded as the dynamical homologue of alignment in communication, which rests upon an amodal representation of a narrative (that can be used for speaking or listening respectively).

This account of communication thus provides us with a theoretical backdrop about the mechanisms that lead to between-brain neural coupling. Furthermore, it makes a specific causal prediction: the extent to which brain activity of speaker and listener are coupled should be modulated by the extent to which the listener has correctly inferred the generative model of the speaker, and thus can predict upcoming input. Between-brain neural coupling can therefore be operationalized as a measure of alignment of the speaker and listener's generative models. However, what remains unclear is what would be represented in such a dynamic generative model, and at what level predictions are made. Based on behavioral research, others have proposed that for a hierarchical system like language, interlocutors align at many different representational levels (Garrod and Pickering, 2009; Pickering and Garrod, 2004), ranging from very low-level acoustic features such as speech rate (Webb, 1969) or accent (Giles and Powesland, 1975), to higher linguistic levels such as the lexical (Brennan and Clark, 1996) and syntactic (Branigan et al., 2000) levels, all with the ultimate goal to align extra-linguistic levels such as the representation of the situation under discussion (i.e. situation model). We hypothesize that the generative model entails all these levels, but interlocutors may be more or less aligned at different levels of the hierarchy.

In line with this hypothesis, we predict that the level of representation on which listeners are aligned with their partner should be reflected in the spatial pattern of between-brain neural coupling. For example, if listeners have aligned their representations with the speaker's at the syntactic level, this should minimally be reflected by neural coupling in cortical areas associated with syntactic processing. Although this hypothesis would definitely require further testing, there is one two-brain study that provides initial evidence. Above, based on the theoretical framework by Friston and Frith (2015a, 2015b), we hypothesized that speaker-listener neural coupling reflects alignment of their generative models, leading to similar predictions about upcoming information. A study by Dikker et al. (2014) measured brain activation (fMRI BOLD response) for one speaker and nine listeners. The speaker described pictures depicting events that could be described with a sentence containing a transitive verb. The lexical-semantic content of the speaker's sentences was classified as predictable or unpredictable, based on the degree to which the items in the depicted scene predicted for specific lexical choices. Predictability was assessed in a separate behavioral experiment. A picture was classified as highly predictable when there was high inter-speaker agreement $(>85 \%)$ in the lexical-semantic content of the sentences used to describe a picture (e.g. a penguin hugging a star: more than $85 \%$ of the speakers described this scene with "the penguin is hugging the star"). For low-predictability items, inter-speaker agreement was low $(<35 \%$, e.g. the guitar is boiling/cooking/stirring the wheel/tire/bike). It is important to note that the predictability of syntactic structure did not vary: the speaker always used simple declarative sentences. The speaker's descriptions were then presented to the listeners. Crucially, Dikker et al. report stronger speaker-listener coupling for predictable relative to unpredictable descriptions in the left posterior superior temporal gyrus, which is, according to them, 
associated with lexical-semantic processing. This study provides initial evidence that speaker-listener neural coupling is influenced by the extent to which the listener is able to predict the speaker's utterance, as would be predicted by our hypothesis. Furthermore, when manipulating predictability at the lexical-semantic level, this leads to variations in coupling in brain areas associated with lexicalsemantic processing.

It would be very interesting if we could extend and test this idea to higher levels of representational alignment. If so, between-brain neural coupling might be an interpersonal neural marker for the ultimate goal of communication: mutual understanding, or alignment at the level of the situation model. We will elaborate on this idea in Section 4 below.

\section{Towards an inter-personal marker of mutual understanding?}

An initial attempt to investigate the relationship between intersubject correlations in brain activity and mutual understanding was done by Stephens et al. (2010). As explained in Section 2 of this paper, there was one speaker telling a story, and eleven listeners who listened to that story in the MRI scanner. What was not mentioned in Section 2 was that after hearing the story, listeners were asked to retell the story that they heard with as much detail as possible. Based on this retelling, Stephens et al. calculated for each listener to what extent the story told by the speaker was successfully communicated (i.e. speaker-listener alignment at the level of the situation model). Successful communication was thus defined as the level of specificity with which listeners could retell the story. This measure was then used as a factor to explain variance in the extent (i.e. the number of brain areas in which significant coupling was found) that the listener's brain activity reflected the speaker's brain activity. Stephens et al. found a positive relationship between their measure of communicative success and the extent of speakerlistener between-brain coupling, which they argue to be evidence in favor of the idea that between-brain neural coupling reflects alignment at the level of the situation model.

However, it should be clear that this study cannot provide conclusive evidence that variations in interpersonal correlations in brain activity reflect variations in alignment at the level of the situation model. Next to the fact that a replication of these results would be warranted, one could question whether this design is best to address the question. If alignment at the level of the situation model is reflected in between-brain neural coupling, we would expect that this type of alignment is independent of the communicative signal. The design used by Stephens et al. does not disentangle neural coupling due to alignment at low levels of linguistic processing, which would depend on the actual communicative signal, from higher, abstract levels at the level of the situation model. Indeed, the same communicative intent could be signaled in many different ways. To extract between-brain coupling due to alignment at the level of situation models, one might compare between-brain coupling for speaker-listener pairs in which the speakers always convey the same communicative intent (e.g. they want to describe an event), but vary in the way they describe that event.

An additional important problem with the study by Stephens et al. is that listeners were asked to retell the story that they had just heard. This assumes that alignment of generative models is a static end-state of a communicative process. However, in their theoretical framework, Friston and Frith assume that the generative model driving predictions, and therefore between-brain coupling, is dynamic and changes over the course of the interaction. A similar idea has been proposed by Stolk et al. (2016). In their conceptual alignment framework, they argue that as the interaction unfolds, communicators continuously update their conceptual spaces (the conceptualization of which we believe to be similar to our earlier conceptualization of a generative model at the level of the situation model). Based on this idea, they predict that not only should producing and interpreting a communicative signal lead to inter-communicator between-brain neural coupling, the temporal dynamics of this shared pattern of neural activity should reflect communicators' adjustments of their shared conceptual spaces (i.e. situation model), which would be crucial for mutual understanding.

Interestingly, they provide support for this hypothesis in a non-verbal communicative hyperscanning fMRI experiment (Stolk et al., 2014). In this experiment, participant pairs were presented with novel and known communicative problems. Crucially, for the novel communicative problems, there was no previously established solution: participants had to coordinate and mutually adjust their situation models or conceptual spaces. Interestingly, Stolk et al. report stronger between-brain neural coupling (in right superior temporal gyrus) when both participants had to adjust their situation model relative to when no such adjustments were necessary. Although Stolk et al. made use of a non-verbal communication paradigm, this may be extended to a verbal communication paradigm in which interlocutors do or do not have to mutually adjust their generative model.

When thinking about between-brain neural coupling as a potential marker for mutual understanding, mutual understanding should not be conceptualized as a static end-state that is the result of successful communication. Rather, it has been argued that speaker-listener neural coupling reflects a continuous process of between-participant alignment of their generative models (Friston and Frith, 2015a, 2015b; Stolk et al., 2016), which, in turn, would be crucial for communication to be successful.

\section{Discussion}

Recently, it has been argued that to study the neural basis of social interaction, which necessarily only exists between individuals, one should not study within-individual brain activity but instead focus on the dynamical interplay between the brains of individuals in interaction. Although this idea is intuitively appealing, it remains unclear what we can learn from such a two-brain approach to interaction: what is reflected in between-brain neural coupling? In the current paper, we zoomed in on the questions that can be addressed by applying the two-brain approach to the study of the neural basis of verbal communication, linking theoretically-motivated frameworks to testable hypotheses and existing empirical data.

In Section 2, we discussed how the hyperscanning method has been used to identify brain networks that are associated with language production as well as language comprehension. Indeed, if activity in area $\mathrm{X}$ in the brain of the sender is temporally correlated with activity in area $X$ in the brain of the listener, this indicates that area $\mathrm{X}$ is associated with encoding as well as decoding of information. Although most (verbal) communication studies have applied this reasoning to study the neural correlates of information transfer, we argued that by focusing on what areas show consistent coupling across all speaker-listener pairs, we ignore possibly valuable information that is represented in between-pair variation at the level of between-brain neural coupling. Therefore, in Section 3, we discussed a theoretical framework (Friston and Frith, 2015a, 2015b) and formulated the hypothesis that the level of betweenbrain coupling depends on how aligned listeners are with a speaker, at different levels of linguistic and extra-linguistic representation. This hypothesis led to the intuitively appealing idea that betweenbrain neural coupling could be an inter-personal neural marker for the highest level of alignment: alignment at the level of the situation model, or mutual understanding. In Section 4, we argued that 
to address this question, alignment at this level should not be conceptualized as a static end-state of communication, but rather as a dynamic and continuous process, which may indeed be reflected in between-brain neural coupling.

Before concluding, there is one last issue we want to address. Almost all two-brain studies on verbal communication that have been discussed so far in this paper have considered communication as a unidirectional process which can be described as transferring information from speaker to listener. In line with this idea, experimental paradigms include two participants, where one is always the speaker (or sender) and one is always the listener (or receiver). In other words, the set-up resembles a monologue (i.e. giving a speech or a lecture) rather than a dialogue, in which participants take turns speaking and listening. Whereas the monologue set-up suffices to investigate whether there is a shared neural circuitry underlying language production and comprehension, it is not the ideal set-up to study inter-personal neural markers of betweensubject alignment. Indeed, we need to consider the fact that alignment of situation models is often the result of a joint process: interlocutors build up meaning together. The Interactive Alignment theory (Pickering and Garrod, 2004), for example, suggests that alignment of situation models is facilitated when interlocutors align their behavioral output (e.g. on the lexical or syntactic level). If between-brain neural coupling is associated with alignment of situation models, an interesting question may be whether aligning behavior results in stronger neural coupling between interlocutors. Initial evidence that supports this idea comes from a study by Jiang et al. (2012), who actually did include a dialogue condition in their two-brain study. Using fNIRS hyperscanning, Jiang et al. investigated neural coupling between interlocutors in dialogue and monologue contexts. They found a significant increase in betweenbrain coupling in the left inferior frontal cortex for face-to-face dialogue, but not for monologue, and attribute their result to the fact that in face-to-face dialogue, there was alignment on different levels of (verbal) behavior and turn-taking, which was not the case for monologue. Although the study by Jiang et al. was not designed to test theoretically-driven, causal predictions like the predictions proposed in this paper, at the very least, their study proves the feasibility of measuring between-brain neural coupling in an interactive, bidirectional setting which resembles natural interaction in dialogue. Together with the causal predictions that have been formulated in the current paper, we argue that the time has come to move to a two-brain approach of verbal interaction, rather than a two-brain approach of one-way verbal communication.

\section{Conclusion}

In this paper, we have addressed the question: what can we learn from a two-brain approach to verbal communication? Although the idea is intuitively appealing, to date, there has been a severe lack of theoretically-driven hypotheses about what between-brain neural coupling actually reflects. We believe that such hypotheses are necessary for the field to move forward. By linking theoretically-motivated frameworks to existing empirical data, we have identified testable hypotheses that may be explored in future research.

\section{Acknowledgments}

We would like to thank Amie Fairs and two anonymous reviewers for their feedback, constructive comments and the valuable additions to our paper.

\section{References}

Anders, S., Heinzle, J., Weiskopf, N., Ethofer, T., Haynes, J.-D., 2011. Flow of affective information between communicating brains. Neuroimage 54, 439-446, http:// dx.doi.org/10.1016/j.neuroimage.2010.07.004.

Babiloni, F., Astolfi, L., 2014. Social neuroscience and hyperscanning techniques: past, present and future. Neurosci. Biobehav. Rev. 44, 76-93, http://dx.doi.org/ 10.1016/j.neubiorev.2012.07.006, Applied Neuroscience: Models, methods, theories, reviews. A Society of Applied Neuroscience (SAN) special issue.

Branigan, H.P., Pickering, M.J., Cleland, A.A., 2000. Syntactic co-ordination in dialogue. Cognition 75, B13-B25, http://dx.doi.org/10.1016/S00100277(99)00081-5.

Brennan, S.E., Clark, H.H., 1996. Conceptual pacts and lexical choice in conversation. J. Exp. Psychol. Learn. Mem. Cogn. 22, 1482-1493, http://dx.doi. org/10.1037/0278-7393.22.6.1482.

Caramazza, A., Zurif, E.B., 1976. Dissociation of algorithmic and heuristic processes in language comprehension: evidence from aphasia. Brain Lang. 3, 572-582, http://dx.doi.org/10.1016/0093-934X(76)90048-1.

Dikker, S., Silbert, L.J., Hasson, U., Zevin, J.D., 2014. On the same wavelength: predictable language enhances speaker-listener brain-to-brain synchrony in posterior superior temporal gyrus. J. Neurosci. 34, 6267-6272, http://dx.doi. org/10.1523/JNEUROSCI.3796-13.2014.

Dumas, G., Lachat, F., Martinerie, J., Nadel, J., George, N., 2011. From social behaviour to brain synchronization: review and perspectives in hyperscanning. IRBM 32, 48-53, http://dx.doi.org/10.1016/j.irbm.2011.01.002 (NUMÉRO SPÉCIAL: LE CERVEAU DANS TOUS SES ÉTATS).

Friston, K., Frith, C., 2015a. A duet for one. Conscious. Cogn. 36, 390-405, http://dx. doi.org/10.1016/j.concog.2014.12.003.

Friston, K.J., Frith, C.D., 2015b. Active inference, communication and hermeneutics Cortex 68, 129-143, http://dx.doi.org/10.1016/j.cortex.2015.03.025 (Special issue: prediction in speech and language processing).

Garrod, S., Pickering, M.J., 2009. Joint action interactive alignment, and dialog. Top. Cogn. Sci. 1, 292-304, http://dx.doi.org/10.1111/j.1756-8765.2009.01020.x.

Giles, H., Powesland, P.F., 1975. Speech Style and Social Evaluation. Academic Press Oxford, England.

Hari, R., Henriksson, L., Malinen, S., Parkkonen, L., 2015. Centrality of social interaction in human brain function. Neuron $88,181-193$, http://dx.doi.org/10. 1016/j.neuron.2015.09.022.

Hasson, U., Ghazanfar, A.A., Galantucci, B., Garrod, S., Keysers, C., 2012. Brain-to-brain coupling: a mechanism for creating and sharing a social world. Trends Cogn. Sci. 16, 114-121, http://dx.doi.org/10.1016/j.tics.2011.12.007.

Jiang, J., Dai, B., Peng, D., Zhu, C., Liu, L., Lu, C., 2012. Neural synchronization during face-to-face communication. J. Neurosci. 32, 16064-16069, http://dx.doi.org/ 10.1523/JNEUROSCI.2926-12.2012.

Konvalinka, I., Roepstorff, A., 2012. The two-brain approach: how can mutually interacting brains teach us something about social interaction? Front. Hum. Neurosci. 6, http://dx.doi.org/10.3389/fnhum.2012.00215.

Kuhlen, A.K., Allefeld, C., Haynes, J.-D., 2012. Content-specific coordination of listeners' to speakers' EEG during communication. Front. Hum. Neurosci., 6, http://dx.doi.org/10.3389/fnhum.2012.00266.

Menenti, L., Garrod, S.C., Pickering, M.J., 2012. Toward a neural basis of interactive alignment in conversation. Front. Hum. Neurosci. 6, 185, http://dx.doi.org/10. 3389/fnhum.2012.00185.

Menenti, L., Gierhan, S.M.E., Segaert, K., Hagoort, P., 2011. Shared language overlap and segregation of the neuronal infrastructure for speaking and listening revealed by functional MRI. Psychol. Sci. 22, 1173-1182, http://dx.doi.org/10. $1177 / 0956797611418347$.

Ménoret, M., Varnet, L., Fargier, R., Cheylus, A., Curie, A., des Portes, V., Nazir, T.A., Paulignan, Y., 2014. Neural correlates of non-verbal social interactions: a dual-EEG study. Neuropsychologia 55, 85-97, http://dx.doi.org/10.1016/j. neuropsychologia.2013.10.001 (Special Issue in Honor of Marc Jeannerod).

Montague, P.R., Berns, G.S., Cohen, J.D., McClure, S.M., Pagnoni, G., Dhamala, M., Wiest, M.C., Karpov, I., King, R.D., Apple, N., Fisher, R.E., 2002. Hyperscanning: simultaneous fMRI during linked social interactions. NeuroImage 16, 1159-1164, http://dx.doi.org/10.1006/nimg.2002.1150.

Pickering, M.J., Garrod, S., 2014. Neural integration of language production and comprehension. Proc. Natl. Acad. Sci. U. S. A. 111, 15291-15292, http://dx.doi. org/10.1073/pnas.1417917111.

Pickering, M.J., Garrod, S., 2004. Toward a mechanistic psychology of dialogue. Behav. Brain Sci. 27, 169-226, http://dx.doi.org/10.1017/S0140525x04000056.

Schippers, M.B., Roebroeck, A., Renken, R., Nanetti, L., Keysers, C., 2010. Mapping the information flow from one brain to another during gestural communication. Proc. Natl. Acad. Sci. U. S. A. 107, 9388-9393, http://dx.doi. org/10.1073/pnas.1001791107.

Segaert, K., Menenti, L., Weber, K., Petersson, K.M., Hagoort, P., 2012. Shared syntax in language production and language comprehension-an fMRI study. Cereb. Cortex 22, 1662-1670, http://dx.doi.org/10.1093/cercor/bhr249.

Silbert, L.J., Honey, C.J., Simony, E., Poeppel, D., Hasson, U., 2014. Coupled neural systems underlie the production and comprehension of naturalistic narrative speech. Proc. Natl. Acad. Sci. 111, E4687-E4696, http://dx.doi.org/10.1073/ pnas.1323812111.

Spiegelhalder, K., Ohlendorf, S., Regen, W., Feige, B., Tebartz van Elst, L., Weiller, C., Hennig, J., Berger, M., Tüscher, O., 2014. Interindividual synchronization of brain activity during live verbal communication. Behav. Brain Res. 258, 75-79, http://dx.doi.org/10.1016/j.bbr.2013.10.015. 
Stephens, G.J., Silbert, L.J., Hasson, U., 2010. Speaker-listener neural coupling underlies successful communication. Proc. Natl. Acad. Sci. 107, 14425-14430, http://dx.doi.org/10.1073/pnas.1008662107.

Stolk, A., Noordzij, M.L., Verhagen, L., Volman, I., Schoffelen, J.-M., Oostenveld, R Hagoort, P., Toni, I., 2014. Cerebral coherence between communicators marks the emergence of meaning. Proc. Natl. Acad. Sci. U. S. A. 111, 18183-18188, http://dx.doi.org/10.1073/pnas.1414886111.
Stolk, A., Verhagen, L., Toni, I., 2016. Conceptual alignment: how brains achieve mutual understanding. Trends Cogn. Sci. 20, 180-191, http://dx.doi.org/10. 1016/j.tics.2015.11.007.

Webb, J.T., 1969. Subject speech rates as a function of interviewer behaviour. Lang. Speech 12, 54-67, http://dx.doi.org/10.1177/002383096901200105. 